\title{
Suppressed Krüppel-like factor 17 expression induces tumor proliferation, metastasis and a poor prognosis in papillary thyroid carcinoma
}

\author{
WEN-CHUN YE ${ }^{1}$, LI GAO ${ }^{1}$, JING HUANG ${ }^{1}$, XIANG-MING FANG ${ }^{1}$ and GANG XIE ${ }^{2}$ \\ Departments of ${ }^{1}$ Endocrinology and ${ }^{2}$ Pathology, Mianyang Central Hospital, Mianyang, Sichuan 621000, P.R. China
}

Received November 20, 2013; Accepted June 5, 2014

DOI: $10.3892 / \mathrm{mmr} .2014 .2429$

\begin{abstract}
Although the role of krüppel-like factor 17 (KLF17) in the regulation of proliferation and epithelial-mesenchymal transition has been examined in breast and liver cancer, their effect on papillary thyroid carcinoma (PTC) remains to be elucidated. The present study aimed to investigate the expression pattern of KLF17 in PTC and the correlation between KLF17 expression and the malignant potential of PTC. KLF17 expression in PTC and adjacent liver tissues was studied by polymerase chain reaction and western blot analysis, and the association between KLF17 expression and the clinicopathological features of PTC was studied in 50 patients. By using RNA interference against KLF17, the correlation between KLF17 expression and malignant potential was examined by downregulating KLF17 expression in TPC-1 cells, and the effects of KLF17 downregulation on cell proliferation and motility were analyzed. Furthermore, the association between KLF17 expression and the surgical outcomes of PTC patients were analyzed. Downregulated expression of KLF17 was associated with a shorter overall survival time in clinical patients $(\mathrm{P}<0.05)$. Low KLF17 expression was significantly associated with tumor stage, tumor size, nodal stage and metastasis stage in PTC $(\mathrm{P}<0.05)$. The reduced expression of KLF17 promoted the motility and proliferation ability of TPC-1 cells by altering the expression of tight junction protein 1 and Snail, and activating the AKT pathway by upregulating inhibitor of DNA binding 1 . In conclusion, the present study demonstrated that KLF17 is important in tumor proliferation and may be a useful
\end{abstract}

Correspondence to: Dr Xiang-Ming Fang, Department of Endocrinology, Mianyang Central Hospital, 12 Jingzhong Street, Mianyang, Sichuan 621000, P.R. China

E-mail: fuyao7688@qq.com

Dr Gang Xie, Department of Pathology, Mianyang Central Hospital, 12 Jingzhong Street, Mianyang, Sichuan 621000, P.R. China E-mail:deantian@sohu.com

Key words: papillary thyroid carcinoma, KLF17, prognosis, proliferation, EMT prognostic indicator in directing therapy. Therefore, further investigation regarding the role of KLF17 in PTC is required.

\section{Introduction}

Thyroid carcinoma accounts for $\sim 1 \%$ of all new malignant diseases, $\sim 0.5 \%$ of cancers in males and $1.5 \%$ in females $(1,2)$. Papillary thyroid carcinoma (PTC) is the most common endocrine malignancy in thyroid carcinoma (3). Although there are numerous pathways associated with this type of tumor, the extracellular signal-regulated kinase/mitogen-activated protein kinase pathway (4) and signal transducer and activator of transcription 3 pathway (5) have been extensively studied. However, at present, more factors need to be identified in order to predict the genesis and development of PTC more precisely. In particular, certain upstream regulators require further examination.

Kruppel-like factors (KLFs) consist of subfamilies belonging to the mammalian specificity protein/KLF zinc-finger protein family (6). Due to their DNA-binding domains, they are important in cancer, inflammation and heart disease (7) through their combination with promoters of target genes in transcription (8).

KLF17 was found to have transactivation activity in embryonic chickens (9) and humans (8). The decrease in the expression of KLF17 has been associated with a poor prognosis and pathological parameters in liver (10) and breast cancer (11). The mechanisms underlying its function include regulation of proliferation and epithelial-mesenchymal transition (EMT) by directly binding to the promoters of inhibitor of DNA binding-1 (Id-1), ZO-1, E-cadherin and Snai1 $(11,12)$. However, the effects of KLF17 on PTC have not been fully elucidated.

The present study explored the correlation between KLF17 expression and malignant clinical-pathological parameters and a poor prognosis in patients with PTC. Cells were transfected with small interfering (si)RNA against KLF17 and their mobility, proliferation ability and expression of Id-1, ZO-1 and Snail were studied. The results indicated that KLF17 may be important in the therapy and prognosis of PTC and thus, further investigation is required.

\section{Materials and methods}

Patient information and samples. In total, 50 pairs of PTC samples and adjacent normal tissues (located $1.5 \mathrm{~cm}$ away from the cancer margin) were collected from patients 
from The General Surgery Department, Mianyang Central Hospital, (Mianyang, Sichuan, China) between March and October 2007. The medical records were obtained at the same time. All samples were cut into $0.5-\mathrm{cm}$ sections and stored at $-80^{\circ} \mathrm{C}$. The clinical parameters, particularly the five-year survival time and outcomes, were gathered through the present study and the follow-up. The KLF17 expression levels between paired tumor and healthy adjacent tissues were examined in all the samples by western blot analysis. The samples whose KLF17 expression in carcinoma tissue was lower than that in adjacent normal tissues were selected as the low KLF17 expression group and vice versa. All the patients underwent surgery to resect the tumor successfully. Pathological data of patients were summarized according to the Union for International Cancer Control (UICC) diagnostic criteria (13). The maximum tumor dimensions were calculated following surgery. Following surgery, the patients were treated with systemic curative radioactive iodine $131+$ thyroid hormone therapy treatment. During the follow-up, serum examination and ultrasound were used to monitor recurrence and metastases. Written informed consent from patients and ethical approval from the Institutional Research Ethics Committee of The Mianyang Central Hospital (Mianyang, China) was obtained prior to initiation of the present study. All clinical information on all patients is summarized in Table I.

Cell lines, cell culture and transient transfection. The 8505C and human PTC (TPC-1) cell lines were purchased from The Cell Bank of Type Culture Collection, Chinese Academy of Sciences (Shanghai, China). The cells were cultured in Dulbecco's modified Eagle's medium (DMEM; Gibco-BRL, Carlsbad, CA, USA) supplemented with $10 \%$ fetal bovine serum and penicillin/streptomycin (all from HyClone, Logan, UT, USA) at $37^{\circ} \mathrm{C}$ in a humidified atmosphere with $5 \% \mathrm{CO}_{2}$. To inhibit the expression of KLF17 (Abcam, Cambridge, UK), siRNA (Guangzhou RiboBio Co., Ltd., Guangzhou, China) was used to transiently transfect the TPC-1 cell line. The transfection was performed using Lipofectamine ${ }^{\mathrm{TM}} 2000$ (Invitrogen Life Technologies, Carlsbad, CA, USA), according to the manufacturer's instructions. TPC-1 cells were seeded at a density of $5 \times 10^{4}$ per well in a six-well plate. The mRNA expression of KLF17 was measured $48 \mathrm{~h}$ after reaching confluence and, following $72 \mathrm{~h}$, the protein expression was also assessed.

Quantitative polymerase chain reaction ( $q P C R)$. The total RNA from paired PTC tumor samples and adjacent normal tissues was extracted. Following application of TRIzol reagent (Invitrogen Life Technologies), the concentration was measured using a NanoDrop 2000 spectrophotometer (NanoDrop Products, Wilmington, DE, USA). Total RNA $(1 \mu \mathrm{g})$ was used in the reverse transcription reaction. The SYBR qPCR mix TM kit (Toyobo, Osaka, Japan) was used for qPCR. Finally, mRNA expression was detected using an ABI Step one real-time PCR system (Applied Biosystems, Sunnyvale, CA, USA). The process was repeated in the TPC-1 cell line to measure the transfection efficiency of KLF17 siRNA. The following specific primers were used: KLF17, forward 5'-GCTCTGGAGTGCACACCTCTT-3' and reverse 5'-CAGCATCTCTGCGCTGTGA-3'; $\beta$-actin (used as control for amplification), forward
5'-TCGTCCACCGCAAATGCTTCTAG-3' and reverse 5'-ACTGCTGTCACCTTCACCGTTCC-3'.

Western blot analysis. The proteins were extracted from tissues and cells and their concentration was measured. Western blotting was performed with specific primary antibodies against KLF17, Id-1, ZO-1, Snai1, AKT, phosphorylated (p)-AKT (rabbit-anti-human antibody; 1:1,000; Abcam, Cambridge, MA, USA) and GADPH (1:1,000; Abcam), followed by appropriate secondary horseradish peroxidase-conjugated antibodies (1:5,000; Pierce Biotechnology, Inc., Rockford, IL, USA). The protein bands were visualized using an enhanced chemiluminescence detection system (Pierce Biotechnology, Inc.).

Cell proliferation assay. To establish cell growth curves, $5 \times 10^{4}$ cells were seeded in 6 -well plates. The plates were divided into two groups. One group was cultured with DMEM only, while the other group was cultured with DMEM following transfection with siRNA against KLF17. The medium was replaced every 2-3 days. The cell numbers were counted on days three, six and nine following seeding. The assay was repeated three times.

Transwell assay. Transfected TPC-1 cells and control cells were seeded at a density of $5 \times 10^{4}$ on the upper chamber of a Transwell plate (24-well insert, $8 \mu \mathrm{m}$ pore size; Corning Costar, Lowell, MA, USA), coated with Matrigel ${ }^{\circledR}$. The lower chamber was filled with $10 \%$ serum serving as a chemoattractant in DMEM. Following $24 \mathrm{~h}$, the cells that had invaded into the lower chamber were fixed in methanol, stained with crystal violet and counted. The area between scratched cells and the number of cells in the transwell assay were measured by Image Pro Plus 6.0 software (Media Cybernetics, Inc., Rockville, MD, USA).

Statistical analysis. The comparison of survival times between the high KLF17 and low KLF17 expression groups was performed by Kaplan-Meier analysis and the log-rank test. The correlation between all sorts of clinical-pathological parameters and the difference in expression of KLF17 was analyzed using a $\chi^{2}$ test. The mean diameter of tumors between two groups was compared by the Wilcoxon rank sum test. In addition, for analyzing the results of qPCR and western blot analysis as well as the Transwell assay, the Student's t-test was adopted. All the data are expressed as the mean \pm standard deviation. $\mathrm{P}<0.05$ was considered to indicate a statistically significant difference. All experimental data were analyzed using the SPSS statistical software (version 16.0; SPSS, Inc., Chicago, IL, USA).

\section{Results}

Expression of KLF-17 is decreased in tumor tissues. Based on qPCR results, the expression of KLF17 significantly decreased in tumor tissues compared with that in adjacent normal tissues. The expression of KLF17 in one of the adjacent normal tissue samples was set as the reference and the value was $1.641 \pm 0.341$ in tumor tissues. The value was $3.81 \pm 0.148$ in adjacent normal tissues (Fig. 1A and B). The difference was statistically significant $(\mathrm{P}<0.05)$ and the protein expression demonstrated a similar result (Fig. 1C). The majority of the adjacent normal tissues exhibited a higher expression of KLF17. 
Table I. KLF17 expression in papillary thyroid carcinoma and the clinical-pathological parameters of 50 patients.

\begin{tabular}{|c|c|c|c|}
\hline Variable & $\begin{array}{l}\text { Low KLF17 expression } \\
\qquad(n=26)\end{array}$ & $\begin{array}{l}\text { High KLF17 expression } \\
\qquad(\mathrm{n}=24)\end{array}$ & $\chi^{2}$-test \\
\hline Gender & $\begin{array}{c}\text { Male (7) } 26.9 \% \\
\text { Female (19) } 73.1 \%\end{array}$ & $\begin{array}{c}\text { Male (6) } 25 \% \\
\text { Female (18) } 75 \%\end{array}$ & $\mathrm{P}=0.877$ \\
\hline Age, years (n) $\%$ & $\begin{array}{l}\geq 45(9) 34.6 \% \\
<45 \text { (17) } 65.4 \%\end{array}$ & $\begin{array}{l}\geq 45(8) 47.1 \% \\
<45(16) 52.9 \%\end{array}$ & $\mathrm{P}=0.619$ \\
\hline Volume of tumor & $\begin{array}{l}\geq 2 \mathrm{~cm}(17) 65.4 \% \\
<2 \mathrm{~cm}(9) 34.6 \%\end{array}$ & $\begin{array}{l}\geq 2 \mathrm{~cm} \mathrm{(8)} 33.3 \% \\
<2 \mathrm{~cm} \mathrm{(16)} 66.7 \%\end{array}$ & $\mathrm{P}=0.048^{*}$ \\
\hline Differentiation & $\begin{array}{c}\text { High/moderate (11) } 42.3 \% \\
\text { Low (15) } 57.7 \%\end{array}$ & $\begin{array}{c}\text { High/moderate (19) } 79.2 \% \\
\text { Low (5) } 20.8 \%\end{array}$ & $\mathrm{P}=0.018^{*}$ \\
\hline T-stage & $\begin{array}{l}\mathrm{T}_{0}-\mathrm{T}_{1}(11) 42.3 \% \\
\mathrm{~T}_{2}-\mathrm{T}_{4}(15) 57.7 \%\end{array}$ & $\begin{array}{l}\mathrm{T}_{0}-\mathrm{T}_{1}(16) 76.2 \% \\
\mathrm{~T}_{2}-\mathrm{T}_{4}(8) 23.8 \%\end{array}$ & $\mathrm{P}=0.041^{*}$ \\
\hline N-stage & $\begin{array}{l}\mathrm{N}_{0}(13) 50 \% \\
\mathrm{~N}_{1}(13) 50 \%\end{array}$ & $\begin{array}{c}\mathrm{N}_{0}(20) 83.3 \% \\
\mathrm{~N}_{1}(4) 16.7 \%\end{array}$ & $\mathrm{P}=0.029^{*}$ \\
\hline M-stage & $\begin{array}{l}M_{0}(16) 61.5 \% \\
M_{1}(10) 38.5 \%\end{array}$ & $\begin{array}{l}\mathrm{M}_{0}(21) 87.5 \% \\
\mathrm{M}_{1}(3) 12.5 \%\end{array}$ & $\mathrm{P}=0.037^{*}$ \\
\hline
\end{tabular}

${ }^{*} \mathrm{P}<0.05$. T, tumor; N, nodes; M, metastasis; KLF17, krüppel-like factor 17.

A

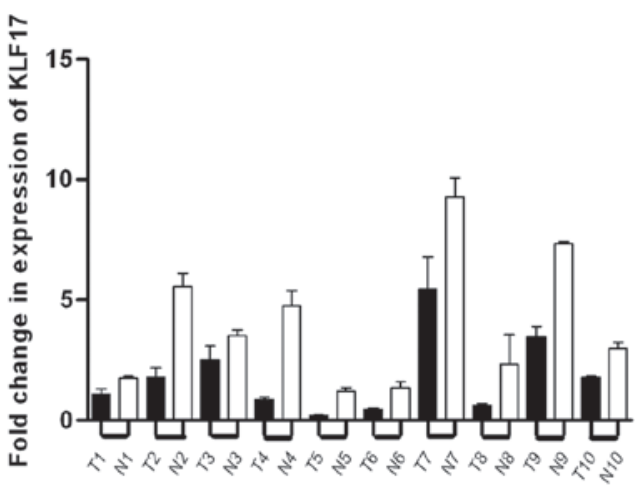

B

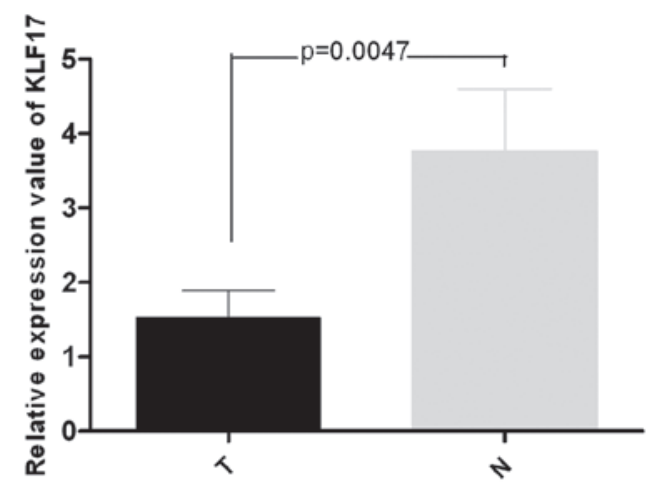

C

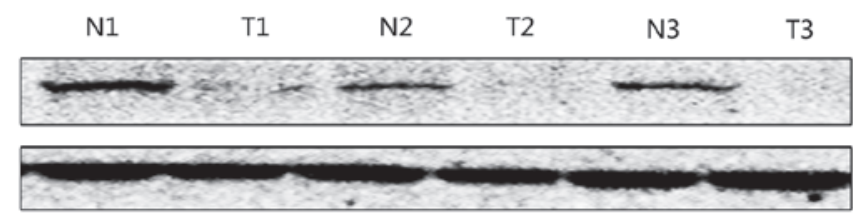

KLF17

GADPH

Figure 1. mRNA and protein expression of KLF17 in tumor tissues is much lower than that in the adjacent normal tissues. (A) Relative mRNA expression of KLF17 in 10 pairs of selected PTC tumors and adjacent normal tissues. One of the normal tissues mRNA expression values was set as one. (B) Mean mRNA expression value of 50 pairs of PTC tumor and adjacent normal tissues. The expression in adjacent normal tissues was significantly higher than that in tumor tissues $(\mathrm{P}<0.05)$. (C) Relative protein expression of KLF17 in three selected pairs of PTC tumor and adjacent normal tissues by western blot analysis. Expression in adjacent normal tissue was significantly higher than that in tumor tissues. KLF17, krüppel-like factor 17; PTC, papillary thyroid carcinoma. T, tumor; $\mathrm{N}$, normal tissue.

KLF17 expression correlates with clinical-pathological parameters and the apparent effects on the prognosis of PTC patients. Next, the $\chi^{2}$ test was adopted to investigate the correlation between KLF17 expression and clinical-pathological data of all patients. The majority of the parameters had no clear association with KLF17 expression (Table I). However, tumor size, T stage, $\mathrm{N}$ stage and $\mathrm{M}$ stage demonstrated a tight correlation with KLF17 expression. The tumors with a dimension $>2 \mathrm{~cm}$ had lower expression levels of KLF17 compared with the group with a tumor dimension $<2 \mathrm{~cm}(\mathrm{P}<0.05$; Fig. $2 \mathrm{~A})$. In addition, the 
A

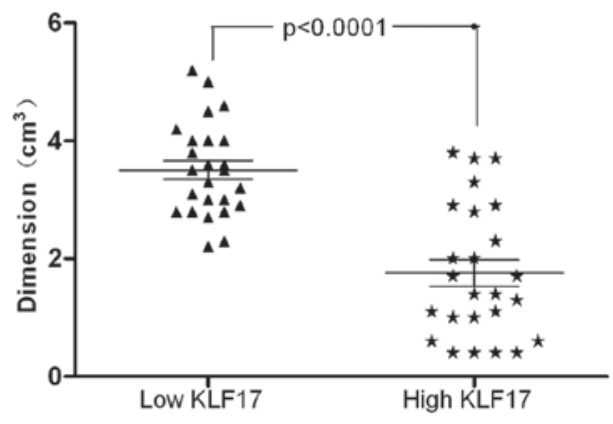

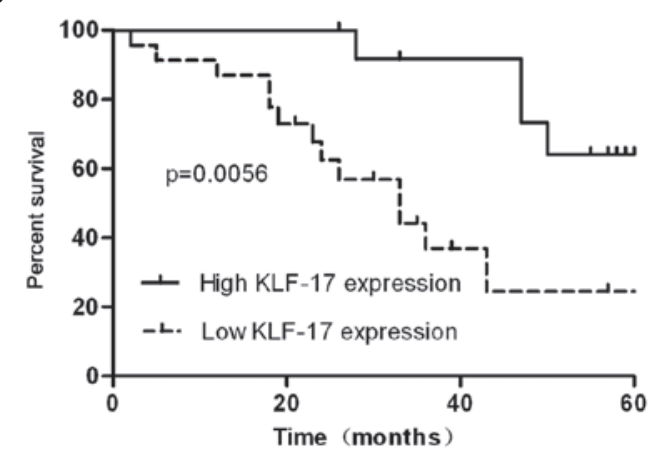

Figure 2. KLF17 affects the pathological parametera and prognosis of PTC patients. (A) Patients with low KLF17 protein expression had a greater dimension of tumors than patients with high KLF17 protein expression. The results were statistically significant (P<0.05). (B) PTC Patients with low KLF17 protein expression had a longer overall survival time than PTC patients with high KLF17 protein expression (P<0.05). KLF17, Krüppel-like factor 17; PTC, papillary thyroid carcinoma.

A

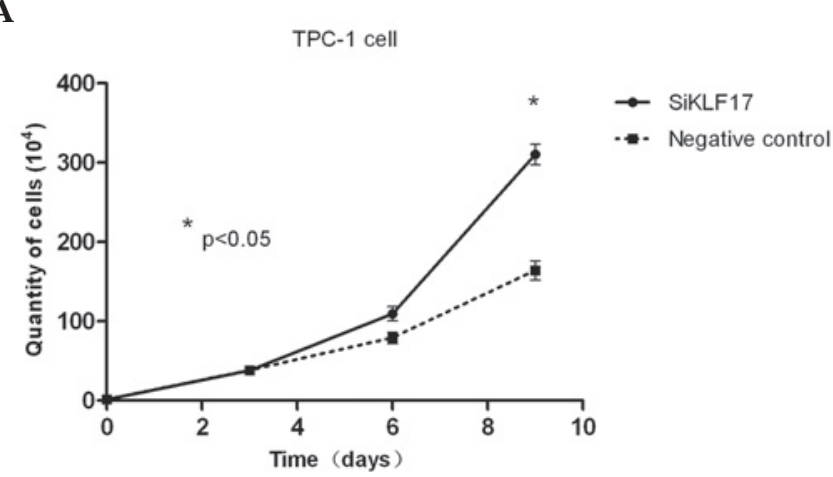

$\mathbf{B}$

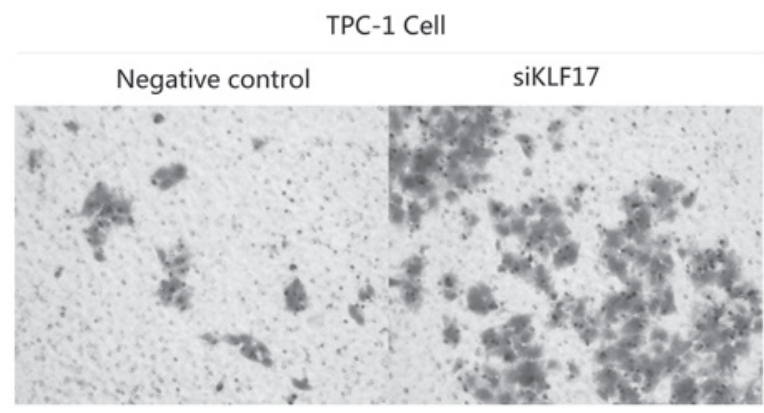

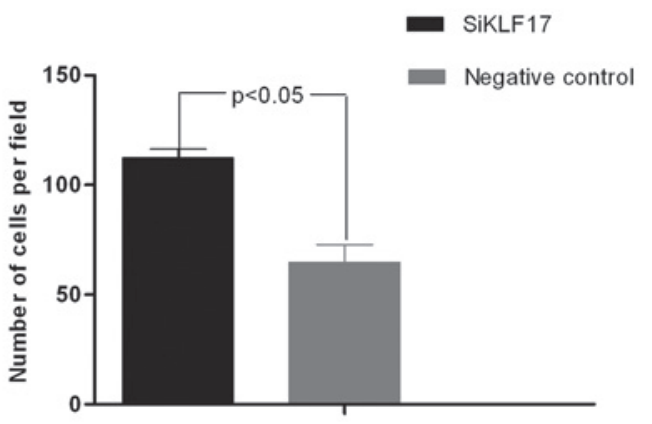

Figure 3. Downregulated KLF17 promotes proliferation and motility in TPC-1 cells. (A) Proliferation assays of TPC-1 cells. The proliferation ability of siKLF17 cells was much higher than that of the negative control cells $(\mathrm{P}<0.05)$. (B) Through the transwell assay, more siKLF17 cells entered the lower chamber than negative control cells. Magnification, $x 40$. The difference in the number of cells was statistically significant $(\mathrm{P}<0.05)$. KLF17, krüppel-like factor 17; siKLF17, siRNA against KLF17.

development of differentiation $(\mathrm{P}=0.018)$, $\mathrm{T}$ stage $(\mathrm{P}=0.041), \mathrm{N}$ stage $(\mathrm{P}=0.029)$ and $\mathrm{M}$ stage $(\mathrm{P}=0.037)$ were negatively correlated with the expression of KLF17.

Patients were then divided into two groups according to their KLF17 expression at the protein level. The survival time of the two groups was compared by Kaplan-Meier analysis. The cumulative five-year survival rate was $64 \%$ in the high KLF17 expression group; however, it was $21 \%$ in the low expression group (Fig. 2B; $\mathrm{P}=0.0073$ ). This finding indicated that downregulated KLF17 expression was associated with a poor prognosis in PTC
Expression of KLF17 affects the proliferation and motility of the PTC cell line. TPC-1 cells were employed as the target of RNA interference (RNAi). siRNA was applied to transfect this cell line to inhibit the expression of KLF17 and the KLF17 mRNA expression levels in these cells were assessed. Following $24 \mathrm{~h}$ transfection, transfected cells and control cells were seeded on 6-well plates. The cell number was determined following three, six and nine days, showing that the proliferation of the transfected group was enhanced, particularly following six days (Fig. 3A; P<0.05). Future studies may aim to identify the correlation between $\mathrm{N}$ and $\mathrm{M}$ stage and KLF17 expression. 
A

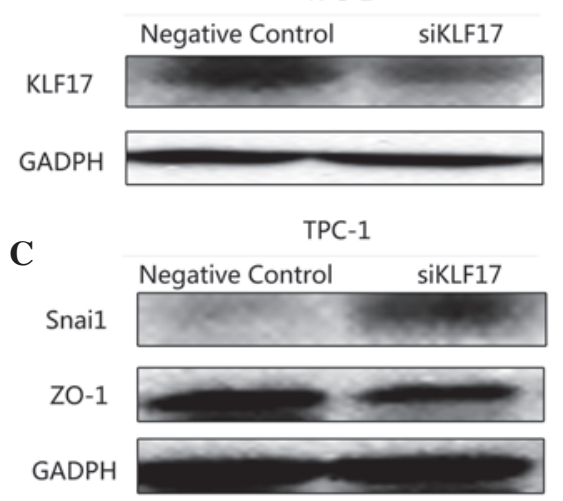

B

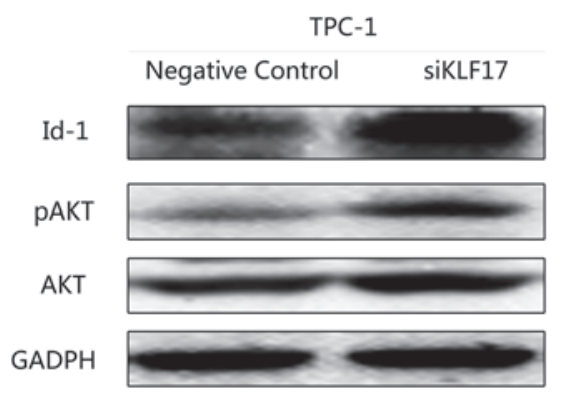

Figure 4. KLF17 promotes proliferation and motility of TPC-1 cells by regulating the expression of the Id-1/AKT pathway, ZO-1 and Snail. Westerm blot analysis revealed the following: (A) Suppressed KLF17 expression was able to increase the expression of Id-1. (B) Suppressed KLF17 expression was able to activate the AKT pathway thus promoting the proliferation of TPC-1 cells. (C) Suppressed KLF17 expression was able to upregulate Snail expression, downregulate ZO-1 expression and induce epithelial-mesenchymal transition. KLF17, Krüppel-like factor 17; Id-1, inhibitor of DNA binding-1; ZO-1, tight junction protein 1.

The invasion ability of transfected TPC-1 cells was also examined. In the Transwell assay, following $24 \mathrm{~h}$, a larger number of cells transfected with siKLF17 penetrated into the lower chamber (Fig. 3B) as compared with the control group. This was statistically significant $(\mathrm{P}<0.05)$.

Inhibition of KLF17 promotes the expression of Id-1, thus activating the AKT pathway and affecting the proliferation of PTC cells. In order to further elucidate the underlying mechanisms of the role of KLF17 in PTC its effect on proliferation, the expression of Id-1 was detected by western blot analysis. With the downregulation of KLF17, Id-1 expression was upregulated (Fig. 4A). AKT and p-AKT were also examined. Consistent with the hypothesis, with the inhibition of KLF17, the AKT pathway was activated (Fig. 4B). This may explain the proliferation changes following transfection.

Inhibition of KLF17 promotes the expression of Snail and suppresses ZO-1, inducing EMT. Next, ZO-1 and Snai1, which have a tight association with EMT, were assessed in transfected TPC-1 cells and control cells by western blot analysis. ZO-1 belongs to markers of epithelial tissues. Snai1 is regarded as a marker of mesenchymal tissues (14). The expression of the two proteins was markedly altered. The expression of ZO-1 was reduced accompanied with an upregulation of Snail in transfected TPC-1 cells (Fig. 4C).

The above findings demonstrated that the downregulation of KLF17 was associated with increased cell motility in TPC-1 cells. It also indicated that the reduction in KLF17 expression was able to induce the occurrence of EMT in PTC, thus, promoting metastasis.

\section{Discussion}

The KLF family consists of several members which each have a particular function in different types of cancer, ranging from oncogenes to tumor suppressors $(15,16)$. KLF17 has been investigated in several types of cancer, including breast cancer, liver cancer and lung adenocarcinoma (17). However, the specific functions of KLF17 remain to be explored in depth and its role in PTC remains to be elucidated.
The present study detected a significantly different expression pattern of KLF17 between tumor tissues and adjacent normal tissues in PTC patients. Furthermore, it was found that tumor dimension and $\mathrm{T}$ staging were negatively correlated with the expression of KLF17. Based on the UICC TNM Classification System for papillary thyroid carcinoma, the majority of patients with lower KLF17 expression were identified at stages 3-4 $(\mathrm{P}<0.05)$. That is possible for the effect of KLF17 on the growth and motility of PTC cells through its effect on Id-1 and EMT-associated factors $(10,11)$. In the low KLF17 expression group, the tumor acquired a stronger metastasis tendency with the invasion of lymph nodes and distant tissues. In addition, prognosis studies have demonstrated that the five-year total survival rate of the patients with low KLF17 expression (40.1\%) was significantly shorter than that of high KLF17 expression patients (60.2\%). In addition, in the high KLF17 expression group, survivors had an improved liver function as compared with the low expression group. These results supported the theory that the KLF17 expression levels are a key prognostic indicator for patients with PTC. KLF17 may not only be an indicator of survival time, but also of quality of life. The present study was, to the best of our knowledge, the first clinical study on PTC demonstrating an apparent clinical correlation between KLF17 expression and poor prognosis.

To verify the results, PTC cell lines were used to compare their KLF17 expression. Through comparison, the $8505 \mathrm{C}$ cell line was found to exhibit a higher KLF17 expression, while the TPC-1 cell line had a lower KLF17 expression. Therefore, TPC-1 cells were selected to inhibit KLF17 expression by RNAi. Following transfection, the expression of KLF17 was verified by western blot analysis. In addition, a cell proliferation assay was applied between control cells and transfected cells. Following $144 \mathrm{~h}$, the proliferation of transfected cells was significantly suppressed compared with that of the control cells $(\mathrm{P}<0.05)$. Based on the Transwell assay, the enhanced potency of invasion and motility in transfected TPC-1 cells verified the function of KLF17 in this area. Next, western blot analysis was employed, showing that with the decrease in KLF17 expression, the expression of Id-1, ZO-1 and Snai1 was altered. Id-1 is a key regulator of tissue-specific gene expression in a number of mammalian and non-mammalian 
organisms, and the constitutive expression of Id proteins has been demonstrated to inhibit the differentiation of various tissues (18). Furthermore, Id-1 exerts its function by promoting the AKT pathway (19). The downregulation of AKT and p-AKT verified our hypothesis. Therefore, it was considered that KLF17 was able to suppress Id-1 expression and thus inhibit the AKT pathway in PTC. Although this result provided a partial explanation for the change in cell proliferation ability, the motility of cells following transfection requires further investigation. The expression of ZO-1 (20) and Snail (21), two typical markers of EMT, were compared between two cell groups. The EMT process (22) affects cell-intrinsic and cell-extrinsic properties of tumor cells by breaking the stability of the cytoskeleton, loosening intercellular junctions and promoting cancer cells to change their form and motility (23). In transfected TPC-1 cells, the expression of ZO-1, a marker of epithelial tissues, was downregulated significantly. In addition, Snai1, a marker for mesenchymal tissue, was markedly upregulated $(\mathrm{P}<0.05)$. KLF17 was linked to the promoter regions of these two genes and shown to affect the expression of the associated proteins (12). Therefore, the expression changes of the two proteins may explain the enhancement in potential for motility and invasion of transfected cells. This may explain the association between low KLF17 expression and poor prognosis of patients with PTC.

In conclusion, the present study demonstrated that KLF17 is a suppressor of PTC growth in preclinical models, although it remains to be determined whether this finding is the case for all histological subtypes of thyroid cancer. However, the present study suggested that targeting this transcription factor may be useful in estimating the prognosis of patients with PTC. As a candidate biomarker, further examining the roles of KLF17 in the progression of PTC and the underlying mechanisms may provide a new therapeutic target to prolong survival time and even enhance the quality of life of PTC patients.

\section{References}

1. De Jong SA: Thyroid cancer: A comprehensive guide to clinical management. Arch Pathol Lab Med 124: 1391, 2000.

2. Sherman SI: Thyroid carcinoma. Lancet 361: 501-511, 2003.

3. DeLellis RA: Pathology and genetics of thyroid carcinoma. J Surg Oncol 94: 662-669, 2006.

4. Fagin JA and Mitsiades N: Molecular pathology of thyroid cancer: diagnostic and clinical implications. Best Pract Res Clin Endocrinol Metab 22: 955-969, 2008.
5. Couto JP, Daly L, Almeida A, et al: STAT3 negatively regulates thyroid tumorigenesis. Proc Natl Acad Sci USA 109: E2361-E2370, 2012

6. Suske G, Bruford E and Philipsen S: Mammalian SP/KLF transcription factors: bring in the family. Genomics 85: 551-556, 2005.

7. McConnell BB and Yang VW: Mammalian Krüppel-like factors in health and diseases. Physiol Rev 90: 1337-1381, 2010.

8. van Vliet J, Crofts LA, Quinlan KG, Czolij R, Perkins AC and Crossley M: Human KLF17 is a new member of the Sp/KLF family of transcription factors. Genomics 87: 474-482, 2006.

9. Antin PB, Pier M, Sesepasara T, Yatskievych TA and Darnell DK: Embryonic expression of the chicken Krüppel-like (KLF) transcription factor gene family. Dev Dyn 239: 1879-1887, 2010.

10. Liu FY, Deng YL, Li Y, et al: Down-regulated KLF17 expression is associated with tumor invasion and poor prognosis in hepatocellular carcinoma. Med Oncol 30: 425, 2013.

11. Gumireddy K, Li A, Gimotty PA, et al: KLF17 is a negative regulator of epithelial-mesenchymal transition and metastasis in breast cancer. Nat Cell Biol 11: 1297-1304, 2009.

12. Sun Z, Han Q, Zhou N, et al: MicroRNA-9 enhances migration and invasion through KLF17 in hepatocellular carcinoma. Mol Oncol 7: 884-94 2013.

13. Sobin LH, Gospodrowicz MK and Wittekind $\mathrm{CH}$ (eds). TNM Classification of Malignant Tumours. 7 th edition. Wiley-Blackwell, New York, NY, 2010.

14. Rowe RG, Li XY, Hu Y, et al: Mesenchymal cells reactivate Snaill expression to drive three-dimensional invasion programs. J Cell Biol 184: 399-408, 2009.

15. Rowland BD and Peeper DS: KLF4, p21 and context-dependent opposing forces in cancer. Nat Rev Cancer 6: 11-23, 2006.

16. Carlson CM, Endrizzi BT, Wu J, et al: Kruppel-like factor 2 regulates thymocyte and T-cell migration. Nature 442: 299-302, 2006.

17. Cai XD, Zhou YB, Huang LX, et al: Reduced expression of Krüppel-like factor 17 is related to tumor growth and poor prognosis in lung adenocarcinoma. Biochem Biophys Res Commun 418: 67-73, 2012.

18. Fong S, Itahana Y, Sumida T, et al: Id-1 as a molecular target in therapy for breast cancer cell invasion and metastasis. Proc Natl Acad Sci USA 100: 13543-13548, 2003.

19. Zhao J, Wang S, Liu N and Tang X: Correlation between the expression of Id-1 and hyperthermia-associated molecules in oral squamous cell carcinoma. J Clin Pathol 66: 758-763, 2013.

20. Umeda K, Ikenouchi J, Katahira-Tayama S, et al: ZO-1 and ZO-2 independently determine where claudins are polymerized in tight-junction strand formation. Cell 126: 741-754, 2006.

21. Rowe RG, Li XY, Hu Y, et al: Mesenchymal cells reactivate Snaill expression to drive three-dimensional invasion programs. J Cell Biol 184: 399-408, 2009.

22. Mani SA, Guo W, Liao MJ, et al: The epithelial-mesenchymal transition generates cells with properties of stem cells. Cell 133: 704-715, 2008.

23. Ishiyama N, Lee SH, Liu S, et al: Dynamic and static interactions between p120 catenin and E-cadherin regulate the stability of cell-cell adhesion. Cell 141: 117-128, 2010. 